\title{
BMJ Open Addressing multiple modifiable risks through structured community-based Learning Clubs to improve maternal and infant health and infant development in rural Vietnam: protocol for a parallel group cluster randomised controlled trial
}

Jane Fisher, ${ }^{1}$ Tuan Tran, ${ }^{2}$ Stanley Luchters, ${ }^{1,3,4}$ Thach D Tran, ${ }^{1,2}$ David B Hipgrave, ${ }^{5,6}$ Sarah Hanieh, ${ }^{7}$ Ha Tran, ${ }^{2}$ Julie Simpson, ${ }^{6}$ Trang Nguyen, ${ }^{1,2}$ Minh Le, ${ }^{1}$ Beverley-Ann Biggs ${ }^{7}$

To cite: Fisher J, Tran T, Luchters S, et al. Addressing multiple modifiable risks through structured community-based Learning Clubs to improve maternal and infant health and infant development in rural Vietnam: protocol for a parallel group cluster randomised controlled trial. BMJ Open 2018;8:e23539. doi:10.1136/ bmjopen-2018-023539

- Prepublication history for this paper is available online. To view these files please visit the journal online (http://dx.doi org/10.1136/bmjopen-2018023539).

Received 15 April 2018 Revised 20 April 2018 Accepted 23 April 2018

Check for updates

For numbered affiliations see end of article.

Correspondence to Professor Jane Fisher; jane.fisher@monash.edu

\section{ABSTRACT}

Introduction Optimal early childhood development is an international priority. Risks during pregnancy and early childhood have lasting effects because growth is rapid. We will test whether a complex intervention addressing multiple modifiable risks: maternal nutrition, mental health, parenting capabilities, infant health and development and gender-based violence, is effective in reducing deficient cognitive development among children aged two in rural Vietnam.

Methods and analysis The Learning Clubs intervention is a structured programme combining perinatal stagespecific information, learning activities and social support. It comprises 20 modules, in 19 accessible, facilitated groups for women at a community centre and one home visit. Evidence-informed content is from interventions to address each risk tested in randomised controlled trials in other resource-constrained settings. Content has been translated and culturally adapted for Vietnam and acceptability and feasibility established in pilot testing. We will conduct a two-arm parallel-group cluster-randomised controlled trial, with the commune as clustering unit. An independent statistician will select 84/112 communes in Ha Nam Province and randomly assign 42 to the control arm providing usual care and 42 to the intervention arm. In total, 1008 pregnant women (12 per commune) from 84 clusters are needed to detect a difference in the primary outcome (Bayley Scales of Infant and Toddler Development Cognitive Score $<1$ SD below standardised norm for 2 years of age) of $15 \%$ in the control and $8 \%$ in the intervention arms, with $80 \%$ power, significance 0.05 and intracluster correlation coefficient 0.03 .

Ethics and dissemination Monash University Human Research Ethics Committee (Certificate Number 20160683), Melbourne, Victoria, Australia and the Institutional Review Board of the Hanoi School of Public Health (Certificate Number 017-377IDD- YTCC), Hanoi, Vietnam have approved the trial. Results will be
Strengths and limitations of this study

This is the first trial to address eight risks to the early development of children in a multicomponent intervention in a resource-constrained setting.

- The intervention is informed by comprehensive local evidence, a long-standing international collaboration among a multidisciplinary group of bicultural investigators and detailed cross-national protocols for implementation and governance.

- The sample size is large so that the trial is powered to detect even small clinically relevant effect sizes.

- Structural equation modelling, an advanced statistical technique, is to be used and will allow us to identify the mechanisms of the trial effects.

- The Bayley Scales of Infant and Toddler Development, the measure of the primary outcome, has not been formally validated against a paediatric developmental assessment in Vietnam.

disseminated through a comprehensive multistranded dissemination strategy including peer-reviewed publications, national and international conference presentations, seminars and technical and lay language reports.

Trial registration number ACTRN12617000442303; Preresults.

\section{INTRODUCTION}

Human development is governed by complex interactions among biological, psychosocial and environmental factors; risks accrue among those in lower socioeconomic positions. $^{12}$ The first 2 years of life are vital as general and neurological growth, reflected in 
cognitive, physical and social-emotional domains, is very rapid and risks during pregnancy and early childhood have lasting effects.

The major risks to optimal child development in resource-constrained settings are:

Anaemia, most commonly caused by iron deficiency poses a significant risk to morbidity and mortality, particularly among women who are pregnant and their children. Higher rates of premature birth and low birth weight occur among women with anaemia in pregnancy. ${ }^{3}$ Up to $50 \%$ of children aged $0-3$ years are anaemic in resource-constrained settings. There is clear evidence that infants with iron deficiency anaemia have worse cognitive development as well as poorer motor, social and/or emotional functioning than those without it. ${ }^{1}$ The long-term benefits of antenatal iron supplementation for child survival and development are not yet well defined. ${ }^{5}$ However, in 1999-2001, 4926 pregnant women in rural Nepal participated in a cluster-randomised, double-masked, controlled trial involving four alternative combinations of micronutrient supplements. Follow-up of their offspring revealed that antenatal iron-folic acid supplementation reduced mortality among $0-7$ year olds by $31 \% .^{6}$ Intellectual functioning was improved by antenatal micronutrient supplementation, ${ }^{6}$ suggesting potential profound effects on child health and development.

Iodine is an essential nutrient and a constituent of the thyroid hormones thyroxine (T4) and triiodothyronine (T3). Globally, 35\% of people have insufficient iodine intake, most because they subsist by agriculture in areas with leached soil. Iodine deficiency disorders adversely affect human growth and development. Among children, iodine deficiency disorders are associated with cognitive and developmental deficits, the most severe being the pervasive developmental disorder of cretinism. Children's IQ scores are on average 13.5 points lower in iodine-deficient than iodine-sufficient areas. ${ }^{7}$

Intrauterine growth restriction (IUGR) is due mainly to macronutritional and micronutritional deficiencies and co-incidental infections during pregnancy that lead to impaired fetal growth and low birth weight $(<2.5 \mathrm{~kg})$. The associations between IUGR and child development are apparent in low-income and high-income countries. Infants of low birth weight in Brazil, ${ }^{8}$ Guatemala ${ }^{9}$ and Jamaica ${ }^{10}$ had worse cognitive and motor development at 12 months than those with average birth weight. Follow-up of the 1970 British Birth Cohort found significant and clinically relevant decrements in academic and professional attainment among adults who had been born small for gestational age compared with adults who had normal birth weight. ${ }^{11}$

Growth potential is similar in all children, and stunting and low weight for age are governed more by environmental than genetic factors. ${ }^{12}$ Stunting, classified as height-for-age $\mathrm{Z}$ score less than $-2 \mathrm{SD}$ from the reference mean, ${ }^{13}$ is observed in nearly a third of children aged $\leq 5$ years in resource-constrained settings. It is an indicator of chronic undernutrition, often compounded by infectious diseases. Stunting at age 24-36 months is associated with subsequent lower cognitive abilities, ${ }^{14}$ worse school performance, premature withdrawal from education, ${ }^{15}$ and lower economic productivity, ${ }^{16}$ even with adjustment for socioeconomic factors. The two periods of greatest vulnerability to stunting are during (1) intrauterine development and (2) the transition from reliance on breastmilk to solid foods, usually in the second 6 months of life. ${ }^{17}$ In early childhood, being underweight and stunted is associated with apathy, less positive affect, less participation in play, more insecure infant-to-parent emotional attachment and, at school age, poorer conduct, attention and social skills ${ }^{15}$ and increased risk of chronic diseases in adulthood. ${ }^{18}$

Children reared in violent neighbourhoods have worse developmental outcomes and higher rates of emotional and behavioural problems than those in safe circumstances. ${ }^{19}$ In resource-constrained settings, family violence is associated with higher rates of death among young children, ${ }^{20}$ particularly girls, but there is as yet limited evidence about impact on child development. In high-income countries, children exposed to family violence have worse cognitive and socioemotional development. ${ }^{21}$

The common mental disorders (CMDs) of depression and anxiety are prevalent among women who are pregnant or have recently given birth. ${ }^{22}$ In high-income countries, there is increasing evidence that prenatal anxiety has an adverse impact on fetal neurodevelopment, heightens risk of premature birth and is associated with cognitive, behavioural and emotional problems in infants. ${ }^{23}$ In resource-constrained settings, the situation is more complex. In Pakistan, when socioeconomic status and maternal body mass index were controlled, the relative risk of low birth weight was 1.9 (95\% CI 1.3 to 2.9) among infants of depressed mothers compared with those of non-depressed mothers, but micronutrient deficiencies were not assessed.

Poor maternal psychological state can lead to neglect or inaccurate interpretation of infant cues, developmentally inappropriate expectations and hostile or inconsistent responses. After childbirth in high-income countries, interactions with primary caregivers influence the infant's, cognitive, emotional and social development. There is less evidence from resource-constrained settings ${ }^{24}$ but maternal depression has been linked directly to higher rates of diarrhoeal and respiratory diseases, stunting and hospital admissions among infants and lower completion of recommended immunisation schedules and worse social-emotional development among young children. ${ }^{2}$

Lack of early learning opportunities and insensitive caregiverchild interactions contribute to loss of developmental potential. Learning opportunities that facilitate early cognitive development include caregiver responsiveness and access to materials that promote age-appropriate language and problem-solving skills. ${ }^{2}$ Infants in resource-constrained settings given additional early cognitive stimulation or learning opportunities have consistently higher subsequent cognitive functioning, task orientation, social 
behaviour and self-confidence than children who did not have early stimulation. ${ }^{25}$ Effective caregivers observe the child's cues, interpreting what these indicate, and respond consistently, contingently and effectively. ${ }^{26}$ Caregivers who are positive, sensitive and responsive and do not use harsh physical punishment facilitate early social-emotional development, ${ }^{2}$ secure infant-to-parent emotional attachment ${ }^{27}$ and higher infant cognitive ability. ${ }^{28}$

\section{Evidence from our research about maternal and infant health in Vietnam}

Vietnam, a lower-middle income country in the South East Asian Region, has a population of $\sim 88$ million people; $74 \%$ live in rural areas, most by subsistence agriculture. The average national annual per capita income in 2016 was USD 2100, but there are rural-urban income disparities with lower per capita incomes in rural provinces. The World Bank estimates that $21.5 \%$ of the population, almost all in rural areas, live in absolute poverty with an individual daily income $<$ USD $1.25 .{ }^{29}$ Vietnam is currently rated 115th out of 188 countries on the Human Development Index: a composite score of average life expectancy, years of schooling and per capita income. ${ }^{29}$

Over the past 15 years, we have built a collaborative research programme to develop capacity and generate local evidence about the psychological and general health of women including during pregnancy and after giving birth and the health and development of young children. We have established the prevalence and determinants of perinatal CMDs among women in the north and the south of Vietnam and among urban and rural groups, ${ }^{3031}$ the course of perinatal depression ${ }^{32}$ and the impact of CMD on preventive healthcare participation. ${ }^{31}$ We have translated, validated and published a suite of psychometric measures for local use. ${ }^{33}{ }^{34}$ We have established the prevalence of anaemia, hookworm infection and deficiencies in iron, folate and vitamins B12 and A among women of reproductive age and shown that iron stores can be replenished with population-based de-worming and iron-folic acid supplementation delivered through routine health services. ${ }^{35} 36$

We have completed a cohort study in the proposed study area. In total 497/523 women who were pregnant were recruited from 50 randomly selected rural communes. They were assessed in early and late pregnancy and, with their babies, at 8 and 26 weeks postpartum. We found that in pregnancy, $32 \%$ of women reported food insecurity, $20 \%$ had a body mass index $<18.5,80 \%$ were iodine deficient, $17 \%$ had iron deficiency anaemia, $19 \%$ had experienced intimate partner violence and a third met criteria for a CMD at least once. $^{30}$ 37-39 All were poor with annual per capita incomes below USD 800. There are no services for perinatal mental healthcare or violent victimisation. These biological, psychological and social risks interact and lead to adverse maternal outcomes during pregnancy and birth and, via direct and indirect mechanisms, to compromised fetal and infant health and development. ${ }^{303140-42}$ The preterm birth rate (14\%) was double that in high-income countries. ${ }^{42}$ We found that risks interact: the poorest women and those who had experienced intimate partner violence were at higher risk of CMD and, when other factors were controlled, women with pregnancy CMD were less likely to use the recommended iodised salt and iron supplements. ${ }^{38}$

In this study, we also revealed risks to the development of 6-month-old infants. In path analyses, we showed that Bayley Scales of Infantand Toddler Development (BSID) cognitive scores were predicted directly by persistent antenatal iron deficiency anaemia (estimated mean difference of -11.62 points; $95 \%$ CI -23.01 to -0.22 ) and antenatal CMD at any point $(-4.80$ points; $95 \%$ CI -9.40 to -0.20$)$; motor scores were predicted directly by antenatal anaemia during late pregnancy $(-2.61$ points, $95 \% \mathrm{CI}-0.57$ to -4.65$)$ and CMD in early pregnancy $(-7.13$ points, $95 \% \mathrm{CI}-3.13$ to -11.13$)$ and social-emotional scores were predicted indirectly by antenatal CMD $(-1.11,95 \%$ CI -1.79 to -0.42$)$ via postnatal CMD, low confidence and less affectionate caregiving. ${ }^{40-42}$

The adverse effects of maternal perinatal CMD on early childhood growth were also established. ${ }^{43}$ When other factors were controlled, children whose mothers experienced CMD in late pregnancy or early postpartum had lower length-for-age $\mathrm{Z}$ score at 15 months of age $(-0.15$, $95 \% \mathrm{CI}-0.28$ to -0.05$)$. It is the first study worldwide to show that father's behaviour is also relevant to child growth as a more hostile marital relationship was associated indirectly with lower toddler length-for-age $\mathrm{Z}$ scores via maternal CMD.

We then conducted an randomised controlled trial (RCT) of antenatal micronutrient supplementation in the same rural province and ascertained that intermittent iron supplementation was sufficient to prevent maternal anaemia, and infants were of similar birth weights to those born to mothers receiving daily iron supplementation (mean difference (MD) $28 \mathrm{~g}$; 95\% CI -22 to 78$).{ }^{44}$ In a follow-up cohort study of the infants born to women in the trial, we found that lower dose antenatal iron supplementation led to impaired growth (Adjusted MD in HAZ were -0.14 (95\% CI -0.28 to -0.01) and motor development (MD $-2.07,95 \% \mathrm{CI}-4.11$ to -0.03 ) in children at 3 years of age. ${ }^{45}$

\section{Interventions in resource-constrained settings}

Interventions to optimise early childhood development in resource-constrained settings have focused predominantly on improving nutrition and physical growth, either through micronutrient supplementation, macronutrition in refeeding programmes or education about breastfeeding, weaning or responsive feeding. ${ }^{46}$ Some have paired this with cognitive stimulation through education about low-cost play materials. ${ }^{47}$ Others have focused on strengthening the mother-infant relationship and social-emotional development by using behavioural assessment items to demonstrate the baby's capacities to the mother and the reciprocity of their interactions. ${ }^{48}$ 
Interventions for maternal mental health problems in these settings have been effective in reducing symptoms, but the benefits for child health and development have been inconsistent and generally limited. ${ }^{1}$ Some have found improvements in maternal knowledge about the infant's developmental needs and capabilities, others have found improvements in caregiving demonstrated through greater compliance with recommended immunisation schedules and fewer episodes of diarrhoea. ${ }^{49}$ Security of mother-infant emotional attachment was improved in one trial, but with little evidence of benefits for weight and stunting. ${ }^{50} \mathrm{~A}$ recent trial found that an enhanced nutrition intervention had no effect on child cognitive development on its own, but if combined with responsive stimulation activities did lead to improved cognitive capacity. ${ }^{51}$ A combined microfinance and participatory education programme about gender empowerment reduced the prevalence of intimate partner violence, but benefits for mental health or any child outcomes were not assessed. ${ }^{52}$

We have contributed evidence that risks to infant development begin in pregnancy ${ }^{40-42}$ and to the existing knowledge base of co-occurring postbirth risks in resource-constrained settings. ${ }^{12}$ We now propose to test a comprehensive approach to improving women's health and early childhood health and development through addressing multiple modifiable risks, in particular maternal nutrition, mental health, parenting capabilities, infant health and development and gender-based violence and empowerment, both before and after birth is required.

\section{OBJECTIVES}

The primary aim is to assess the effectiveness of an 18-month, 20-module, structured, facilitated women's health and early childhood development Learning Clubs intervention in reducing deficient cognitive development among 2 year olds in rural Vietnam.

It is hypothesised that fewer children whose mothers participated in the Learning Clubs intervention will have Bayley Scales of Infant and Toddler Development cognitive scores $<-1$ SD below age-specific norms at 2 years of age compared with children whose mothers received only usual care.

\section{METHODS AND ANALYSIS Trial design}

The trial is a two-arm parallel group cluster-randomised controlled trial (cRCT) of the Learning Clubs intervention. The intervention is for groups of women and, in order to limit contamination of the usual care services, randomisation of individuals is not possible. The unit of clustering is the commune, which is the primary government entity in Vietnam. Each commune has a local administration, a health centre and a population of $\sim 7-10000$ people. All women will receive the usual standard of pregnancy and postpartum healthcare, including free antenatal checks, birth in a medical facility and access to the National Growth Monitoring and Expanded Immunisation Programmes. In Arm 1, women will receive the Learning Clubs intervention throughout pregnancy (8 modules) and during the first year of the child's life (12 modules, a total of 20 modules) in addition to standard care. In Arm 2, women will receive only the usual standard of care.

\section{Study setting}

The trial will be conducted in Ha Nam, a typical Red River rural province in the North of Vietnam that has sufficient infrastructure to conduct this trial, represents the geographic zone and does not have conflicting studies taking place.

\section{Participants}

Eligibility criteria

All women who are aged at least 18 years, pregnant and less than 20 weeks' gestation (determined on the basis of the first day of the last menstrual period) living in the selected communes will be eligible to participate.

\section{Exclusion criteria}

Women who have a cognitive disability (determined by the local commune health station staff) or other serious physical disabilities which prohibit attendance.

\section{Learning Clubs intervention}

The Learning Clubs intervention is a structured programme that combines perinatal stage-specific essential information, learning activities and social support in accessible facilitated community-based groups of women. The intervention comprises 20 educational modules, delivered in face-to-face groups at a community centre and in one home visit. The evidence-informed content has been drawn from interventions to address individual risks: maternal nutrition, mental health or parenting capabilities, infant health and/or development or gender-based violence and empowerment which have been tested in RCTs in other resource-constrained settings and supplemented by our deep knowledge of local risk factors and health and social protection systems to ensure cultural sensitivity and appropriateness (figure 1). These include the Thinking Healthy, ${ }^{49}$ What Were We Thinking, ${ }^{39}$ Sisters for Life ${ }^{52}$ and Care for Child Development programmes ${ }^{53}$ and WHO guidelines for nutrition and breastfeeding. ${ }^{54}$ Nutritional messages are drawn from WHO guidelines from the e-Library of Evidence for Nutrition Actions and Essential Nutrition Action ${ }^{55}$ and include dietary diversity, the use of supplements and fortified foods and the importance of adequate nutritional intake.

Programme content has been translated, adapted to ensure that it is culturally appropriate for Vietnamese women and field-tested for salience and comprehensibility. All content has been checked for alignment with current national perinatal programmes in Vietnam by the Maternal and Child Health Department of the Ministry of 


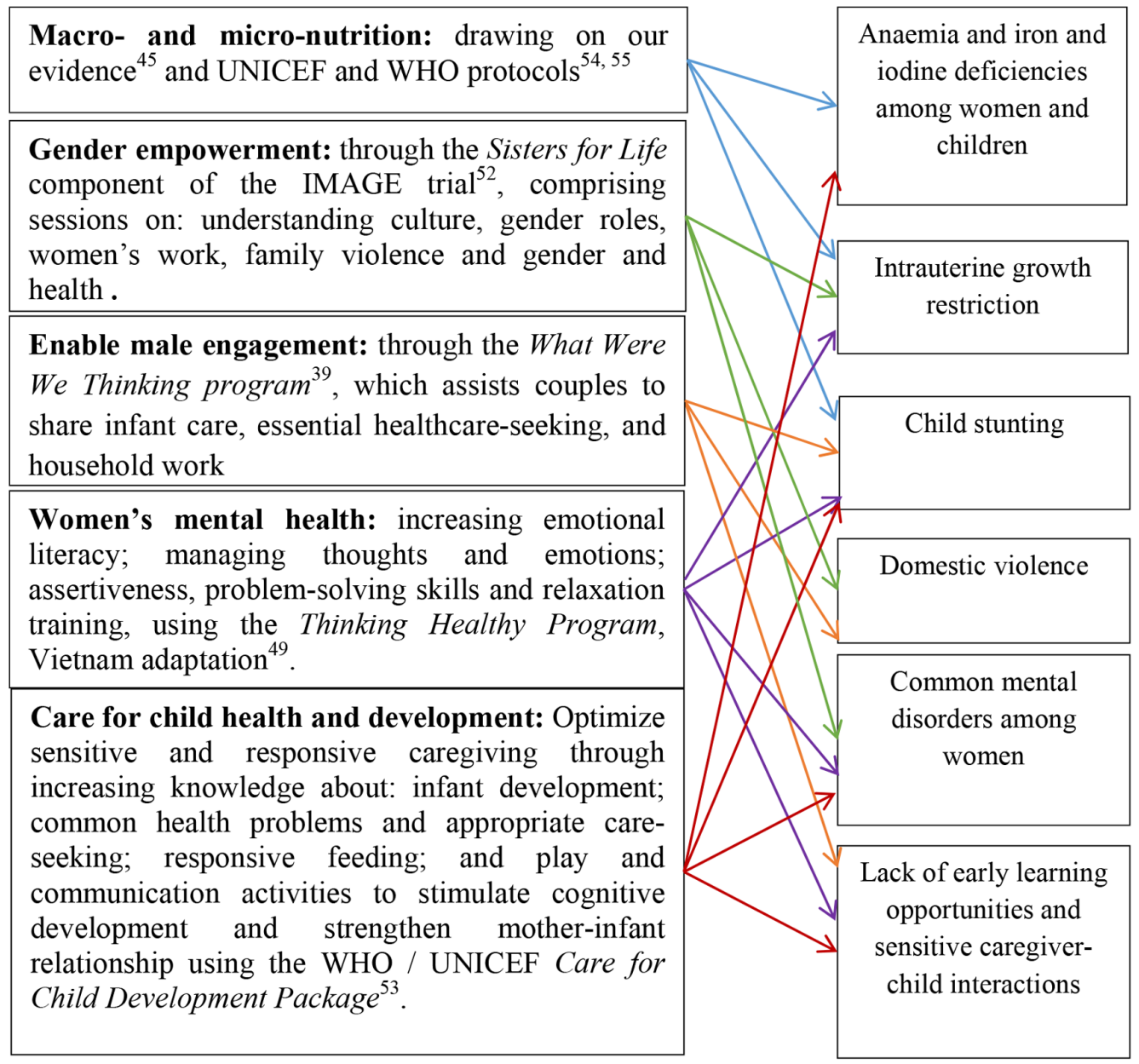

Figure 1 Learning Club content addressing risks to women's health and early childhood development.

Health. The programme has a coherent set of illustrated participant materials suitable for people with low literacy, short DVDs and a facilitator's guide to ensure fidelity.

The programme will be implemented in facilitated small groups of women meeting every 2 weeks in community centres from mid-pregnancy and every 4 weeks after childbirth, until the end of the first postpartum year (a total of 19 facilitated group sessions) and one home visit during the first eight postpartum weeks when mothers and infants in this setting usually do not go out of the house.

Each small group session will be facilitated by a trained and supervised member of the local Women's Union, who has had experience in conducting community programmes and works in one commune only. Vietnam's Women's Union is a highly structured national social organisation, with branches at all levels, including local communes. Its mandate is to engage women in activities related to women's health and the family. When required because of specific content, a community health worker and or a kindergarten teacher will also participate. Each session will be from 60 to $90 \mathrm{~min}$ in duration, with shorter sessions scheduled for the first two postpartum meetings to accommodate infant feeding and sleeping requirements.
Educational information will be given to participants through videos, brief talks and participatory learning activities. These will be varied to maintain interest and will include short quizzes, role-play, problem-solving discussions, physical exercises for pregnancy, breathing techniques for birth, observation of their babies to understand infant capabilities and behavioural cues, demonstrations of caregiving for optimal child development, discussing and solving scenario-based exercises to understand and promote gender empowerment and mental health and prevent gender-based violence. During each session, take-home materials will be distributed to each participant. These materials will provide a summary of the information the participants are given at each session, recommended activities they can do at home and take-home messages. Content is tailored to be stage specific, with core nutrition and mental health content and selected content about empowerment and child care, health and development.

An early postpartum household visit will be conducted by a trained commune health station staff member. The purpose of the visit is to screen for postbirth complications and newborn warning signs and to guide women and their family members on how to soothe and settle the baby 
Table 1 Outcome and mediator indicators and assessment tools

\begin{tabular}{lll}
\hline Variable & Tool & Description \\
\hline Primary outcome & & \\
$\begin{array}{l}\text { Toddler Cognitive } \\
\text { development }\end{array}$ & Bayley Scales of Infant and Toddler & Direct child assessments. We have translated the \\
& Development (BSID), 3rd Ed & Bayley Scales of Infant and Toddler Development into \\
& Cognitive Score & Vietnamese, adapted materials where necessary and \\
& & trained local expert administrators. Reference mean \\
& score is $100(\mathrm{SD}=15)$.
\end{tabular}

\begin{tabular}{|c|c|c|}
\hline \multicolumn{3}{|l|}{ Secondary outcomes } \\
\hline $\begin{array}{l}\text { Infant anthropometric } \\
\text { indices }\end{array}$ & $\begin{array}{l}\text { Mother-infant scale (Seca } 876) \text {; } \\
\text { portable stadiometers and length } \\
\text { boards (ShorrBoard) }\end{array}$ & $\begin{array}{l}\text { Infant height-for-age, weight-for-age and weight-for- } \\
\text { height z scores will be calculated by WHO methods }{ }^{13} \\
\text { using length and weight based on infant's age and sex. }\end{array}$ \\
\hline $\begin{array}{l}\text { Infant Motor and Social- } \\
\text { emotional development }\end{array}$ & $\begin{array}{l}\text { Bayley Scales of Infant and Toddler } \\
\text { Development }\end{array}$ & Direct child assessments. \\
\hline $\begin{array}{l}\text { Infant Cognitive } \\
\text { development }\end{array}$ & $\begin{array}{l}\text { Bayley Scales of Infant and Toddler } \\
\text { Development }\end{array}$ & Direct child assessments. \\
\hline \multicolumn{3}{|l|}{ Mediators } \\
\hline $\begin{array}{l}\text { Home environment for } \\
\text { infant development }\end{array}$ & $\begin{array}{l}\text { Infant/Toddler Home Observation } \\
\text { for Measurement of the Environment } \\
\text { (HOME) Inventory } 60\end{array}$ & $\begin{array}{l}\text { Comprises } 45 \text { items assessing quality and quantity } \\
\text { of stimulation and support available to a child at } \\
\text { home through semistructured observation and parent } \\
\text { interview at home. }\end{array}$ \\
\hline Maternal Hb level & HemoCue 210 & Standardised field measurement. \\
\hline $\begin{array}{l}\text { Maternal urinary iodine } \\
\text { concentration }\end{array}$ & $\begin{array}{l}\text { Analyses at an accredited Vietnamese } \\
\text { laboratory }\end{array}$ & $\begin{array}{l}\text { Urine samples frozen in the field and transported in a } \\
\text { cold chain to a national accredited laboratory. }\end{array}$ \\
\hline Infant $\mathrm{Hb}$ level & HemoCue 210 & Standardised field measurement. \\
\hline
\end{tabular}

effectively and to promote the baby's development through play and communication. The visit is expected to take 45-60 min.

As a whole, the intervention will meet the five pillars recommended by the WHO for programmes to improve maternal and child health: family support, empathic listening, guided discovery using culturally appropriate images and text-based materials, behavioural activation and collaborative problem solving. ${ }^{56}$

\section{Outcomes, mediators and covariates}

Primary and secondary outcomes and potential effect mediators of the trial are described in table 1.

\section{Covariates}

- Quality of parent-parent relationship: measured by the Intimate Bonds Measure, Vietnam Validation ${ }^{34}$ (yielding two subscales: 'Care', assessing sensitivity, empathy and trust and 'Control', assessing criticism, coercion and dominance) and the WHO MultiCountry Study on Women's Health and Domestic Violence Questionnaire. ${ }^{57}$

- Women's reproductive history: assessed by study-specific structured questions about gravidity, parity, history of miscarriage and stillbirth and neonatal deaths.

- Infant birth weight and gestational age at birth: calculated from reported dates of last menstrual period and giving birth. Birth weights from parent-held child health records.

- Sociodemographic characteristics: number of household members, parents' education and occupation assessed by study-specific questions. Household Wealth Index calculated using the World Bank method assessing 17 household characteristics, services and durable assets. ${ }^{1}$

\section{Participant timeline}

In both study arms of the proposed cRCT, baseline data (B) will be collected early in the mid-trimester of pregnancy (12-20 gestational weeks) and there will be three follow-up study visits: in late pregnancy (after 32 weeks of gestation, F1), when the infant is 1year old (F2) and 2years old (F3). Allocation of communes will be conducted at timepoint 0 ; there will be no participant involvement at that stage. The overall participant timeline is described in table 2 . 
Table 2 Participant timeline

\begin{tabular}{|c|c|c|c|c|c|}
\hline \multirow[b]{3}{*}{ Timepoint } & \multicolumn{5}{|l|}{ Study period } \\
\hline & \multirow{2}{*}{$\begin{array}{l}\text { Enrolment of } \\
\text { participants } \\
\text { B }\end{array}$} & \multirow{2}{*}{$\begin{array}{l}\begin{array}{l}\text { Allocation of } \\
\text { clusters }\end{array} \\
0 \\
\end{array}$} & \multicolumn{3}{|l|}{ Postallocation } \\
\hline & & & F1 & $\mathbf{F} 2$ & F3 \\
\hline \multicolumn{6}{|l|}{ Enrolment } \\
\hline Eligibility screen & $x$ & & & & \\
\hline Informed consent & $x$ & & & & \\
\hline Allocation & & $\mathrm{X}$ & & & \\
\hline \multicolumn{6}{|l|}{ Interventions (for those in intervention communes) } \\
\hline \multicolumn{6}{|l|}{ Learning clubs } \\
\hline \multicolumn{6}{|l|}{ Assessments: } \\
\hline \multicolumn{6}{|l|}{ Mother } \\
\hline Maternal common mental disorders & $x$ & & $x$ & $x$ & $x$ \\
\hline Maternal serum ferritin & $x$ & & $x$ & & \\
\hline Maternal haemoglobin level & $x$ & & $x$ & & \\
\hline Maternal urinary iodine concentration & $x$ & & $\mathrm{x}$ & & \\
\hline Quality of parent-parent relationship & $x$ & & $x$ & $x$ & $x$ \\
\hline Mother's reproductive history & $x$ & & & & \\
\hline Sociodemographic characteristics & $x$ & & & & \\
\hline \multicolumn{6}{|l|}{ Child } \\
\hline $\begin{array}{l}\text { Bayley Scales of Infant and Toddler } \\
\text { Development }\end{array}$ & & & & & $x$ \\
\hline Infant anthropometric indices & & & & $\mathrm{x}$ & $x$ \\
\hline Home environment for infant development & & & & $x$ & $x$ \\
\hline Infant haemoglobin level & & & & $\mathrm{x}$ & $x$ \\
\hline Infant microbiome & & & & $x$ & $x$ \\
\hline Infant birth weight and gestational age at birth & & & $\mathrm{x}$ & & \\
\hline
\end{tabular}

B: Baseline; F1: Late pregnancy (32 weeks of gestation); F2: when infant is 1 year old; F3: when toddler is 2 years old.

\section{Sample size and power calculation}

The number of clusters and sample size have been calculated using the clustersampsi module in Stata, V.13 (Stata, College Station, Texas, USA). A total of 1008 pregnant women from 84 clusters (in each study arm: 504 women, $\approx 12$ women per commune (the cluster) recruited from 42 clusters) is necessary to detect a difference in the primary outcome (BSID cognitive development score $<1 \mathrm{SD}$ at 2 years of age) of $15 \%$ in the control arm and $8 \%$ in the Learning Club intervention arm (with $80 \%$ statistical power and a significance level of 0.05 ; intracluster correlation coefficient $=0.03$ ). These assumptions are derived from previous studies in Vietnam ${ }^{41} 4244$ and other resource-constrained settings. ${ }^{5} 58$ This sample size will give us $\geq 85 \%$ power to detect differences between the two arms of the secondary outcomes (table 3). Sample size and power calculations take into account loss to follow-up from still births $(<1 \%)$, late abortions $(<1 \%)$, infant mortality $(0.2 \%)$, relocation $(5 \%)$ and withdrawal $(13 \%)$, estimated to be $\sim 20 \% .^{3}$

\section{Assignment of interventions}

An independent statistician will randomly select 84 communes (unit of clustering) from the lists of a total of 112 communes in Ha Nam province and randomly allocate 42 to each study arm. The randomisations will be conducted using Stata V.14.0. The independent statistician who conducts the randomisation is not involved any other activities of this study.

\section{Recruitment}

It is estimated from our previous studies that 15-25 women will be eligible per cluster. ${ }^{41}{ }^{42}{ }^{44}$ In a process we have used successfully, commune health centre staff and village health workers will identify and approach potential participants to inform them of the study and invite those who are interested to visit the commune health centre. ${ }^{30}$ The recruitment process will begin with 12 pregnant women per commune being randomly-selected from the list of eligible women. Each woman who declines the invitation to participate will be replaced 


\begin{tabular}{lrrr}
\hline \multicolumn{3}{l}{ Table 3} & Power calculations for secondary outcomes \\
\hline & MD* & ICC & Power \\
\hline $\begin{array}{l}\text { Height-for-age Z score at } \\
\text { 2years old }\end{array}$ & 0.25 & 0.03 & 0.93 \\
$\begin{array}{l}\text { Weight-for-age Z score at } \\
\text { 2years old }\end{array}$ & 0.3 & 0.01 & 0.98 \\
$\begin{array}{l}\text { Weight-for-height Z score at } \\
2 \text { years old }\end{array}$ & 0.2 & 0.01 & 0.85 \\
$\begin{array}{l}\text { BSID Cognitive score at 1 year } \\
\text { old }\end{array}$ & 0.25 & 0.02 & 0.94 \\
$\begin{array}{l}\text { BSID Motor score at 2 years } \\
\text { ld }\end{array}$ & 0.45 & 0.01 & 0.96 \\
$\begin{array}{l}\text { BSID Social-emotional score at } \\
\text { 2years old }\end{array}$ & 0.3 & 0.03 & 0.90 \\
\hline
\end{tabular}

*MD: standardised mean difference (SD of every BSID scale $=15$ scale points). MDs and ICCs are derived from our previous studies in Vietnam. ${ }^{414244}$

BSID, Bayley Scales of Infant and Toddler Development; ICC, intracluster correlation coefficient.

by another randomly selected woman until 12 are recruited.

Recruitment of participants will be conducted by representatives of the commune health centre and the local Women's Union, who will not be involved in the randomisation of commune clusters.

\section{Blinding}

Data collectors and data analysts will be blinded to the assignment of communes to trial arms. All data collectors will interview participants at baseline, Follow-up 1, 2 and 3 without knowing which trial arm the commune has been assigned to and therefore the participant belongs to. Participants will each be assigned a code number which identifies the commune, but not the trial arm. Trial assignment of each commune will only be made available to data analysts after unblinding at the end of the trial.

\section{Data collection methods}

The completion of self-report questionnaires is an unfamiliar and generally difficult task for people living in rural Vietnam and data collection by structured individual interviews is accepted standard practice. ${ }^{8}$ Assessments of mothers, including study-specific questions and standardised psychometric instruments will be completed by 12 trained supervised health researchers of the Hanoi Research and Training Centre for Community Development (RTCCD).

Infant Cognitive, Motor and Social-Emotional development will be assessed using the Bayley Scales of Infant and Toddler Development (BSID) 3rd Edition. The BSID has been translated into Vietnamese and materials adapted where necessary. ${ }^{40-42}$ We have trained a team of local psychologists to undertake the individual BSID assessments at 12 and 24 months and will maintain quality through periodic observations by the one BSID-accredited trainer in Vietnam.
All interviews and infant development assessments will be conducted in private rooms at local commune health centres.

Maternal urinary iodine concentration will be assessed in casual urine samples of approximately $10 \mathrm{~mL}$ obtained from each participant in late pregnancy. Urine samples will be frozen in a field freezer and transported in a cold chain to the laboratory of the National Hospital of Endocrinology in Hanoi, who have conducted urine iodine concentration (UIC) analyses for many national surveys in Viet Nam. At the laboratory, UIC will be determined by means of the Sandell-Koltho reaction, as recommended by WHO, Unicef and The International Council for the Control of Iodine Deficiency Disorders (ICCIDD). Iodine nutritional status is classified as adequate if $\mathrm{UIC}=150 \mu \mathrm{g} / \mathrm{L}$ ) or deficient if $\mathrm{UIC}<150 \mu \mathrm{g} / \mathrm{L}$ according to the WHO/Unicef/ICCIDD guidelines.

Maternal anaemia will be assessed in the field through maternal haemoglobin ( $\mathrm{Hb})$ concentration from a finger prick blood sample, using a hemoglobinometer (HemoCue AB, Angelholm Sweden). We will classify maternal anaemia according to WHO and Unicef recommendations ( $\mathrm{Hb}$ concentrations $<11 \mathrm{~g} / \mathrm{dL}$ ).

Infant anaemia will be assessed in the field through infant $\mathrm{Hb}$ concentration from a heel prick blood sample, using a hemoglobinometer (HemoCue AB, Angelholm Sweden). According to the recommendations by WHO and Unicef, infants are classified as having anaemia when $\mathrm{Hb}$ concentration $<11 \mathrm{~g} / \mathrm{dL}$.

Maternal iron deficiency will be assessed through maternal serum ferritin. Venous blood samples will be collected from the mother by an experienced phlebotomist from the Vietnam National Institute of Hematology and Blood Transfusion. Serum will be separated by spinning with a field centrifuge and being frozen for transportation to Hanoi, storage at the National Institute at $-20^{\circ} \mathrm{C}$ and, after data collection is complete, transfer to Australia on dry ice. Serum will be analysed for ferritin level by an accredited Australian laboratory. Iron deficiency is defined as serum ferritin level of $<15 \mathrm{ng} / \mathrm{mL}$.

\section{Plans to promote participant retention}

Participation rates at each commune assigned to the intervention arm for each Learning Club session will be closely monitored, by facilitator reports and attendance sheets. Communes in which participation rates fall below $70 \%$ will be followed up and participants in these communes visited by a representative from the local Women's Union to identify any difficulties or obstacles, which prevent participation. Barriers will be addressed where possible.

\section{Data management}

Data will be collected using hand-held computer devices. Range checks will be incorporated into the devices so that out of range values cannot be entered. Data will be uploaded from the hand-held devices to a password protected database on a secure cloud-based storage 
system daily. The database will be built and managed by an experienced data manager at RTCCD. All participants will be assigned a code number and all data will be identified and entered using only code numbers and not names. Data will be checked and cleaned regularly by the RTCCD data manager. Once each wave of data has been collected, it will be transferred to Australia for storage on the secure Monash University server. Data will only be accessible to approved members of the research staff.

\section{Statistical methods}

After descriptive analyses, data will be analysed in three steps. First, the differences in the primary and secondary outcomes between the two arms will be estimated by individual-level analyses adjusting for clustering. Second, mixed-effect logistic regression for the main outcome and mixed-effect linear regression models for the secondary outcomes taking into account covariates if there are baseline differences among the arms and incorporating a random effect for commune (clustering) and a fixed effect for trial arm will be conducted to estimate the effects of the intervention on the outcomes. Third, mediation analyses will be conducted using the structural equation modelling technique. All analyses will follow intention-to-treat principles, applying multiple imputation for missing data and be performed at individual level using Stata, V.14 (Stata, College Station, Texas, USA).

\section{Data monitoring}

Data monitoring will be conducted by an independent Data Monitoring Committee (DMC) including a biostatistician, a paediatrician and a public health physician. The DMC will assess the outcomes, the safety data and overall progress of the trial at follow-up 1 (when the women are in the third trimester) and 6 months after follow-up 1 (when the babies are about 4 months old). Depending on the results, the DMC will provide recommendations to the steering committee whether to continue, modify or stop the trial.

\section{Harms}

All participants (both mothers and infants) will be closely monitored for any International Council for Harmonisation of Technical Requirements for Pharmaceuticals for Human Use - Guideline for Good Clinical Practice ((ICH-GCP) defined adverse events that may arise. At the beginning of each Learning Club session, facilitators will ask participants for any events they may want to report. Any event arising during the session will also be noted. For each event, facilitators will assess to see if it is a medical emergency. If it is, usual procedure in case of an emergency in Vietnam will be followed (dial 115 and paramedic nurses will come to attend to the patient). If not, facilitators will report to the Steering Committee, which is chaired by a paediatrician and includes representatives from the Maternal and Child Health Department, Ministry of Health; the Department for Child Care and Protection, Ministry of Labour, Invalids and Social Affairs and the Federal Women's Union in Vietnam and will then decide whether or not to refer the participant to appropriate support services.

In case of serious adverse events due to trial intervention or trial conduct, the Steering Committee will discuss and decide whether or not to cease the trial. It is thought, given the nature of the intervention, that this is extremely unlikely to happen.

Any woman who becomes distressed because of participation will be referred to appropriate support services. Contact details of these services are provided in the participant information sheet at time of enrolment.

\section{Public involvement}

Over the past 18 years our Vietnam-Australia team has built a collaborative research programme to develop capacity and generate local evidence, about the psychological and general health of women during pregnancy and after giving birth and the health and development of young children in rural Vietnam. All this work has been undertaken in close consultation with the Vietnam Ministry of Health and the Ha Nam Provincial Health Department. In specific aspects, we have collaborated with the Vietnam Medical Association, the National Institute of Nutrition, the National Institute of Endocrinology, the Vietnam Union of Science and Technology Associations and United Nations agencies, in particular the Vietnam offices of the WHO and Unicef and international non-government agencies with aligned interests. In this project, we are working closely with the Vietnam Women's Union which is a highly structured national social organisation, with branches at all social levels, including local communes. Its mandate is to engage women in activities related to women's health and the family. We have held regular symposia in which progress on evidence generation was presented and discussed with all these stakeholders. We then convened roundtable consultations with them about the content and structure of a low-cost, evidence-informed, community based intervention to address the problems we had identified. The programme and the trial design have been carefully considered and formally approved by the Vietnam Ministry of Health Maternal and Child Health Department and the Vietnam Union of Science and Technology Associations.

We pilot-tested the Learning Clubs programme in three communes in Ha Nam province and ascertained acceptability and salience in interviews and small group discussions with women and community members who had participated. The content was in general found to be highly acceptable to participants: 'I felt more confident in taking care of my son and it saved my money from buying formula milk' (A 22-year-old teacher in Doi Son commune). 'The content is very practical. We live in a rural area so we don't have opportunities to learn officially, but only by my grandmother's and my mother's experience. Now with all the knowledge provided by the club I can take better care of my children' (participant from Liem Can Commune). An unexpected outcome was the extraordinarily high community interest in the Learning Clubs 
activities. At closure of the pilot test, 918 people, including 613 women and 305 partners and grandparents, had attended a Learning Clubs session.

We identified aspects of the programme requiring amendment. Women's Union facilitators were confident in providing most content, but said that 'Mental health is a new concept for us and it is uncommon to share our thoughts to others' (a facilitator in Liem Can commune) and 'we are not familiar with making toys and playing with children by ages' (a facilitator in Doi Son commune). These have been revised in the Learning Clubs programme to be assessed in this trial by including commune health workers and kindergarten teachers as cofacilitators of specific sessions.

In designing the trial, participants informed us that the 24 sessions that had been proposed were too many and might interfere with income-generating work. We modified the programme to comprise 20 sessions, one of which is a postbirth home visit.

A Technical Advisory and Policy Advocacy Board of representatives of the Vietnam Ministry of Health, WHO, Unicef, Save the Children Vietnam and other international and local NGOs working in maternal and child health will oversee the project. If the Learning Clubs approach proves effective, the Board will advocate for the inclusion of this innovation in the Essential Health Services, to be covered under the National Universal Insurance Scheme. The Investigators, co-operating with the Board, will use a multistranded dissemination strategy to ensure translation of findings. It will include, seminar and media presentations, national policy briefings, local-language technical reports and lay language summaries to be posted on notice boards and announced on the loudspeaker systems in participating communes in Vietnam.

\section{ETHICS AND DISSEMINATION \\ Protocol amendments}

All modifications to the protocol will be reported to the investigators through regular meetings or emails, ethics committees through amendments application forms or by email if these are not used, trial participants through announcements on commune notice boards and trial registries through the registration portal. The chief investigator with the project manager will take responsibility for ensuring that these are completed.

\section{Consent}

Potential participants will be informed about the study by a commune health centre staff member and invited to visit the commune health centre at a specified time if they are interested to learn more about it. Announcements will also be made on the commune loudspeaker system in order to reach women who might not yet have registered their pregnancy. Participant information packages including a plain language participant information sheet and a consent form will be distributed to women who meet eligibility criteria and express interest in the study. A commune staff member will read the information sheet and consent form to women who are unable to read them. Consent can be given by a signature or witnessed oral consent or for those who are unable to write and wish to, by thumbprint. Contact details of an investigator will be provided if participants have questions or concerns about the trial. Maternal consent will be sought on behalf of the infant for data to be collected about the infant.

\section{Confidentiality}

Participants will be identified on all project documents by individual code numbers and not their names. Data will be entered into a password-protected secure database only by code number and not by name. It will be stored first in a secured folder on a cloud-based secure storage system at RTCCD. Once each wave of data collection is complete, data will be transferred to the Monash University secure server which is backed up daily. Deidentified data will only ever be accessible to authorised members of the research team. Signed consent forms will be stored in a locked filing cabinet at RTCCD. Only pooled data from which no individual could be identified will ever be published.

\section{Access to data}

Data will be entered into password protected files and can only be accessed by authorised personnel, including chief investigators, project manager and research officers and assistants. At trial completion and after publication of the findings, deidentified participant level quantitative survey data will be published online using Figshare, which enables free public access. However, permission to use the data, including for publication purposes, has to be sought from the investigators and the source of the data acknowledged.

\section{Dissemination policy}

We will use a comprehensive multistranded dissemination strategy. It includes peer-reviewed publications, national and international conference presentations, seminars and technical reports. We will engage with print, broadcast and social media in Vietnam and internationally. We will provide national policy briefings in Vietnam, local-language reports and lay language summaries for participants and the commune communities. Every effort will be made to facilitate publication in open access journals.

\section{DISCUSSION}

This is to our knowledge the first trial to address eight risks to the early development of children in a multicomponent intervention in a resource-constrained setting. It places an explicit focus on addressing the health and psychological needs of women who are pregnant or providing primary care for infants and young children and the principle 
that consideration of women's needs and rights requires respectful prioritisation in endeavours to improve the growth and health of children. In this intervention, the benefits to women and their children of freedom from interpersonal violence, valuing of unpaid work and gender empowerment are addressed directly.

The intervention is informed by comprehensive local evidence, a long-standing international collaboration among a multidisciplinary group of bicultural investigators and detailed cross-national protocols for implementation and governance. It has been developed in close collaboration with potential users and stakeholders. Feasibility, acceptability and preliminary evidence of impact have been established in pilot-testing. The intervention and the trial have the support of the national Ministry of Health and the Ha Nam Provincial Department of Health in Vietnam and the approval of all local auspicing authorities.

Nevertheless, there are potential limitations in our approach.

It is predicated on the capacity of community-based workers, who do not have preservice training in the theory and skills that underpin the intervention, to implement it over an 18-month period. We are addressing this by dividing the 20 sessions into five modules and providing training at the beginning of each module, regular supportive supervision and an easy to follow illustrated facilitator's guide with short scripts and step-by-step instructions. Fidelity will be enhanced by the provision of short DVDs to explain or demonstrate session content. We amended the implementation protocol to include the commune health worker to assist with the presentation of mental health content and kindergarten teachers to assist with ways of engaging with and providing cognitive stimulation for babies.

The content has been drawn from English language resources and there is potential that some words or constructs do not have exact linguistic equivalents in Vietnamese. We have addressed this by having all translations undertaken by more than one bilingual research worker, with group discussion to resolve uncertainty about meaning. All participant and facilitator materials have been illustrated by a Vietnamese graphic designer to maximise comprehensibility and demonstrate meaning.

In order to optimise the early development of children from intrauterine life to the end of the first year after birth, the intervention has 20 sessions, each designed to address women's developmental stage specific needs for knowledge and skills. This number of sessions is similar to those established in the Thinking Healthy Program, ${ }^{49}$ and in Walker et $a l$ s trial of cognitive stimulation in Jamaica. ${ }^{10}$ However, participation might not be maintained for the entire programme. We addressed this following the pilot-test, by combining and synthesising some content and reducing the number of sessions from 24 to 20. Groups are offered at two-week (during pregnancy) and four-week (during the postpartum year) intervals to minimise disruption to income-generating work. In order to maximise behaviour change, at each session, core key-messages will be revisited with discussion of how in practice these are being implemented and how further changes might be made.

The primary outcome is being assessed by the BSID, 3rd Ed ${ }^{59}$ Cognitive Score. We have adapted a small set of items to make them recognisable to children (eg, replaced the image of a donkey, which no children will have seen, with one of a water buffalo, which are widespread in the area). However, it has not been possible in this (or any other low-income or middle-income country) to conduct a formal validation against a paediatric developmental assessment because to do this for adequate numbers of children for each month of age requires enormous resources which have not been available.

Last, we have selected a defined set of secondary outcomes and covariates, but it is possible that other unascertained mechanisms might influence the outcomes. Overall however, the data are likely to make a substantial, valuable contribution to knowledge about a low-cost, integrated, universal approach to improving the early development of young children by attending to their needs and, most importantly to the needs of their primary caregivers.

\section{Trial status}

Trial recruitment will start in May 2018 and, at the time of manuscript submission, has not started.

\section{Author affiliations}

${ }^{1}$ School of Public Health and Preventative Medicine, Monash University, Melbourne, Victoria, Australia

${ }^{2}$ Research and Training Centre for Community Development, Hanoi, Vietnam ${ }^{3}$ Centre for International Health, Burnet Institute, Melbourne, Victoria, Australia ${ }^{4}$ International Centre for Reproductive Health, Department of Obstetrics and Gynaecology, Ghent University, Gent, Belgium

${ }^{5}$ New York Headquarters, United Nations Children's Fund, New York City, New York, USA

${ }^{6}$ Melbourne School of Population and Global Health, University of Melbourne, Melbourne, Victoria, Australia

${ }^{7}$ Department of Medicine and Victorian Infectious Diseases Service, Doherty Institute, University of Melbourne, Melbourne, Victoria, Australia

Contributors JF, TDT and ML prepared the first draft. TT, SL, DBH, SH, HT, JS, TN and $B-A B$ reviewed and contributed to revisions. All authors agreed on the content of the final submitted version.

Funding This research is funded by Australian National Health and Medical Research Council Project Grant (GNT1100147) and received seed funding from Grand Challenges Canada under the Saving Brains Initiative (2014-2015). JF is supported by a Monash Professorial Fellowship and the Jean Hailes Professorial Fellowship. TDT and SH are each supported by Australian National Health and Medical Research Council Early Career Research Fellowships. SL is supported by an Australian National Health and Medical Research Council Career Development Fellowship. TN holds an Australian Postgraduate Award. ML holds a Monash University Bridging Postdoctoral Fellowship.

Disclaimer None of the funders have had or will have a role in study design, collection, management, analysis and interpretation of data, writing of reports or decision to submit reports for publication.

Competing interests None declared.

Patient consent Not required.

Ethics approval Monash University Human Research Ethics Committee (Certificate Number 20160683), Melbourne, Victoria, Australia and the Institutional Review Board of the Hanoi School of Public Health (Certificate Number 017-377IDD-YTCC), Hanoi, Vietnam.

Provenance and peer review Not commissioned; peer reviewed for ethical and funding approval prior to submission. 
Data sharing statement At trial completion and after publication of the findings, deidentified participant level quantitative survey data will be published online using Figshare, which enables free public access. However, permission to use the data, including for publication purposes, has to be sought from the investigators and the source of the data acknowledged.

Open access This is an open access article distributed in accordance with the Creative Commons Attribution Non Commercial (CC BY-NC 4.0) license, which permits others to distribute, remix, adapt, build upon this work non-commercially, and license their derivative works on different terms, provided the original work is properly cited and the use is non-commercial. See: http://creativecommons.org/ licenses/by-nc/4.0/

(C) Article author(s) (or their employer(s) unless otherwise stated in the text of the article) 2018. All rights reserved. No commercial use is permitted unless otherwise expressly granted.

\section{REFERENCES}

1. Walker SP, Wachs TD, Gardner JM, et al. Child development: risk factors for adverse outcomes in developing countries. Lancet 2007;369:145-57.

2. Walker SP, Wachs TD, Grantham-McGregor S, et al. Inequality in early childhood: risk and protective factors for early child development. Lancet 2011;378:1325-38.

3. Aukett MA, Parks YA, Scott PH, et al. Treatment with iron increases weight gain and psychomotor development. Arch Dis Child 1986;61:849-57.

4. Grantham-McGregor S, Ani C. A review of studies on the effect of iron deficiency on cognitive development in children. J Nutr 2001;131:649S-68.

5. Pasricha SR, Hayes E, Kalumba K, et al. Effect of daily iron supplementation on health in children aged 4-23 months: a systematic review and meta-analysis of randomised controlled trials. Lancet Glob Health 2013;1:e77-e86.

6. Christian P, Murray-Kolb LE, Khatry SK, et al. Prenatal micronutrient supplementation and intellectual and motor function in early schoolaged children in Nepal. JAMA 2010;304:2716-23.

7. Bleichrodt N, Garcia I, Rubio C, et al. Developmental disorders associated with severe iodine deficiency. In: Hetzel B, ed. The prevention and control of IDD. Amsterdam: Elsevier, 1987:65-84.

8. Grantham-McGregor SM, Lira PI, Ashworth A, et al. The development of low birth weight term infants and the effects of the environment in northeast Brazil. J Pediatr 1998;132:661-6.

9. Gorman KS, Pollitt E. Relationship between weight and body proportionality at birth, growth during the first year of life, and cognitive development at 36, 48, and 60 months. Infant Behavior and Development 1992;15:279-96.

10. Walker SP, Chang SM, Powell CA, et al. Psychosocial intervention improves the development of term low-birth-weight infants. J Nutr 2004;134:1417-23.

11. Strauss RS. Adult functional outcome of those born small for gestational age: twenty-six-year follow-up of the 1970 British Birth Cohort. JAMA 2000;283:625-32.

12. Grantham-McGregor S. Early child development in developing countries. The Lancet 2007;369:824.

13. WHO. Physical status: the use and interpretation of anthropometry. Geneva: WHO, 1995.

14. Walker SP, Chang SM, Powell CA, et al. Effects of early childhood psychosocial stimulation and nutritional supplementation on cognition and education. Lancet 2005;366:1804-7.

15. Chang SM, Walker SP, Grantham-McGregor S, et al. Early childhood stunting and later behaviour and school achievement. J Child Psychol Psychiatry 2002;43:775-83.

16. Black RE, Allen LH, Bhutta ZA, et al. Maternal and child undernutrition: global and regional exposures and health consequences. Lancet 2008;371:243-60.

17. Badham J, Sweet L. Stunting: an overview. Sight and Life 2010;3:40-7.

18. de Onis M, Branca F. Childhood stunting: a global perspective. Matern Child Nutr 2016;12(Suppl 1):12-26.

19. Leventhal T, Brooks-Gunn J. The neighborhoods they live in: the effects of neighborhood residence on child and adolescent outcomes. Psychol Bull 2000;126:309-37.

20. Silverman JG, Decker MR, Cheng DM, et al. Gender-based disparities in infant and child mortality based on maternal exposure to spousal violence. Arch Pediatr Adolesc Med 2011;165:22.

21. Veltman MW, Browne KD. Three decades of child maltreatment research implications for the school years. Trauma, Violence, \& Abuse 2001;2:215-39.
22. Fisher J, Cabral de Mello M, Patel V, et al. Prevalence and determinants of common perinatal mental disorders in women in low- and lower-middle-income countries: a systematic review. Bull World Health Organ 2012;90:139-49.

23. Bergman K, Sarkar P, Glover V, et al. Maternal prenatal cortisol and infant cognitive development: moderation by infant-mother attachment. Biol Psychiatry 2010;67:1026-32.

24. Rahman A, Bunn J, Lovel H, et al. Association between antenatal depression and low birthweight in a developing country. Acta Psychiatr Scand 2007;115:481-6.

25. Watanabe K, Flores R, Fujiwara J, et al. Early childhood development interventions and cognitive development of young children in rural Vietnam. J Nutr 2005;135:1918-25.

26. Eshel N, Daelmans B, de Mello MC, et al. Responsive parenting: interventions and outcomes. Bull World Health Organ 2006;84:991-8.

27. Valenzuela M. Maternal sensitivity in a developing society: the context of urban poverty and infant chronic undernutrition. Dev Psychol 1997;33:845-55.

28. Agarwal DK, Awasthy A, Upadhyay SK, et al. Growth, behavior, development and intelligence in rural children between 1-3 years of life. Indian Pediatr 1992;29:467-80.

29. UNDP. Human Development Report 2010. New York: UNDP, 2010.

30. Fisher J, Tran T, Duc Tran T, et al. Prevalence and risk factors for symptoms of common mental disorders in early and late pregnancy in Vietnamese women: a prospective population-based study. $J$ Affect Disord 2013;146:213-9.

31. Fisher J, Tran T, La BT, et al. Common perinatal mental disorders in northern Viet Nam: community prevalence and health care use. Bull World Health Organ 2010;88:737-45.

32. Fisher JR, Morrow MM, Ngoc NT, et al. Prevalence, nature, severity and correlates of postpartum depressive symptoms in Vietnam. BJOG 2004;111:1353-60.

33. Tran TD, Tran T, Fisher J. Validation of the depression anxiety stress scales (DASS) 21 as a screening instrument for depression and anxiety. BMC Psychiatry 2013;13:24.

34. Fisher J, Tran TD, Biggs B, et al. Validation of the Intimate Bonds Measure for women who are pregnant or have recently given birth in Vietnam. Asia Pac Psychiatry 2014;6:28-37.

35. Casey GJ, Jolley D, Phuc TQ, et al. Long-term weekly iron-folic acid and de-worming is associated with stabilised haemoglobin and increasing iron stores in non-pregnant women in Vietnam. PLoS One 2010;5:e15691.

36. Casey GJ, Tinh TT, Tien NT, et al. Sustained effectiveness of weekly iron-folic acid supplementation and regular deworming over 6 years in women in rural Vietnam. PLoS Negl Trop Dis 2017;11:e0005446.

37. Fisher J, Tran TD, Biggs B, et al. Intimate partner violence and perinatal common mental disorders among women in rural Vietnam. Int Health 2013;5:29-37.

38. Fisher J, Tran T, Biggs B, et al. lodine status in late pregnancy and psychosocial determinants of iodized salt use in rural northern Viet Nam. Bull World Health Organ 2011;89:813-20.

39. Fisher JR, Wynter KH, Rowe HJ. Innovative psycho-educational program to prevent common postpartum mental disorders in primiparous women. BMC public health 2010;10:432.

40. Tran TD, Tran T, Simpson JA, et al. Infant motor development in rural Vietnam and intrauterine exposures to anaemia, iron deficiency and common mental disorders: a prospective community-based study. BMC Pregnancy Childbirth 2014;14:8.

41. Tran TD, Biggs BA, Tran T, et al. Perinatal common mental disorders among women and the social and emotional development of their infants in rural Vietnam. J Affect Disord 2014;160:104-12.

42. Tran TD, Biggs BA, Tran T, et al. Impact on infants' cognitive development of antenatal exposure to iron deficiency disorder and common mental disorders. PLoS One 2013;8:e74876.

43. Fisher J, Tran T, Nguyen T, et al. Common mental disorders among women, social circumstances and toddler growth in rural Vietnam. Child Care Health Dev 2015;41:843-52.

44. Hanieh S, Ha TT, Simpson JA, et al. The effect of intermittent antenatal iron supplementation on maternal and infant outcomes in rural Viet Nam: a cluster randomised trial. PLoS Med 2013;10:e1001470

45. Hanieh S, Ha TT, Simpson JA, et al. Effect of low-dose versus higherdose antenatal iron supplementation on child health outcomes at 36 months of age in Viet Nam: longitudinal follow-up of a cluster randomised controlled trial. BMJ Glob Health 2017;2:e000368.

46. Engle PL, Black MM, Behrman JR, et al. Strategies to avoid the loss of developmental potential in more than 200 million children in the developing world. Lancet 2007;369:229-42.

47. Jin X, Sun Y, Jiang F, et al. "Care for Development" intervention in rural China: a prospective follow-up study. J Dev Behav Pediatr 2007;28:213-8. 
48. Cooper PJ, Tomlinson M, Swartz L, et al. Improving quality of mother-infant relationship and infant attachment in socioeconomically deprived community in South Africa: randomised controlled trial. BMJ 2009;338:b974.

49. Rahman A, Malik A, Sikander S, et al. Cognitive behaviour therapybased intervention by community health workers for mothers with depression and their infants in rural Pakistan: a cluster-randomised controlled trial. Lancet 2008;372:902-.

50. Cooper PJ, Landman M, Tomlinson M, et al. Impact of a motherinfant intervention in an indigent peri-urban South African context: pilot study. Br J Psychiatry 2002;180:76-81.

51. Yousafzai AK, Rasheed MA, Rizvi A, et al. Effect of integrated responsive stimulation and nutrition interventions in the Lady Health Worker programme in Pakistan. Lancet 2014;384:1282.

52. Pronyk PM, Hargreaves JR, Kim JC, et al. Effect of a structural intervention for the prevention of intimate-partner violence and HIV in rural South Africa: a cluster randomised trial. Lancet 2006;368:1973-83.

53. WHO and UNICEF. Care for child development: improving the care of young children. Geneva: World Health Organisation, 2012.
54. WHO \& UNICEF. Infant young child feeding counselling: WHO \& UNICEF, 2006.

55. WHO. e-Library of Evidence for Nutrition Actions (eLENA). Secondary e-Library of Evidence for Nutrition Actions (eLENA). 2017. http:// www.who.int/elena/en/

56. Zafar S, Sikander S, Haq Z, et al. Integrating maternal psychosocial well-being into a child-development intervention: The five-pillars approach, 2014.

57. Garcia-Moreno C, Jansen $\mathrm{H}$, Ellsberg M, et al. WHO multi-country study on women's health and domestic violence against women. Geneva: World Health Organization, 2005.

58. Rahman A, Fisher J, Bower P, et al. Interventions for common perinatal mental disorders in women in low- and middle-income countries: a systematic review and meta-analysis. Bull World Health Organ 2013;91:593-601.

59. Bayley N. Bayley scales of infant and toddler development. 3rd edn. San Antonio: TX: Harcourt Assessment, 2006.

60. Caldwell BM, Bradley RH. Home observation for measurement of the environment. Little Rock: University of Arkansas at Little Rock, 1984. 